\title{
THE TENERIFE COSMIC MICROWAVE BACKGROUND MAPS: OBSERVATIONS AND FIRST ANALYSIS
}

\author{
C. M. Gutiérrez ${ }^{1}$, R. Rebolo ${ }^{1,2}$, R. A. Watson ${ }^{1,3}$, \\ R. D. Davies ${ }^{3}$, A. W. Jones ${ }^{4}$, and A. N. Lasenby ${ }^{4}$
}

Received —; accepted —

\footnotetext{
${ }^{1}$ Instituto de Astrofísica de Canarias, E 38200 La Laguna, Tenerife, Canary Islands, Spain

${ }^{2}$ Consejo Superior de Investigaciones científicas

${ }^{3}$ Mullard Radio Astronomy Observatory, Cavendish Laboratory, Madingley Road, Cambridge CB3 OHE, UK

${ }^{4}$ University of Manchester, Nuffield Radio Astronomy Laboratories, Jodrell Bank, Macclesfield, Cheshire SK11 9DL, UK
} 


\begin{abstract}
The results of the Tenerife Cosmic Microwave Background (CMB) experiments are presented. These observations cover 5000 and 6500 square degrees on the sky at 10 and $15 \mathrm{GHz}$ respectively centred around Dec. $\sim+35^{\circ}$. The experiments are sensitive to multipoles $l=10-30$ which corresponds to the Sachs-Wolfe plateau of the CMB power spectra. The sensitivity of the results are $\sim 31$ and $\sim 12 \mu \mathrm{K}$ at 10 and $15 \mathrm{GHz}$ respectively in a beam-size region ( $5^{\circ} \mathrm{FWHM}$ ). The data at $15 \mathrm{GHz}$ show clear detection of structure at high Galactic latitude; the results at $10 \mathrm{GHz}$ are compatible with these, but at lower significance. A likelihood analysis of the 10 and $15 \mathrm{GHz}$ data at high Galactic latitude, assuming a flat CMB band power spectra gives a signal $\Delta T_{\ell}=30_{-8}^{+10} \mu \mathrm{K}$ (68\% C.L.). Including the possible contaminating effect due to the diffuse Galactic component, the CMB signal is $\Delta T_{\ell}=30_{-11}^{+15} \mu \mathrm{K}$. These values are highly stable against the Galactic cut chosen. Assuming a Harrison-Zeldovich spectrum for the primordial fluctuations, the above values imply an expected quadrupole $Q_{R M S-P S}=20_{-7}^{+10} \mu \mathrm{K}$ which confirms previous results from these experiments, and which are compatible with the COBE DMR data in the case of the standard inflationary Cold Dark Matter models.
\end{abstract}

Subject headings: cosmic microwave background-cosmology: observations 


\section{INTRODUCTION}

In the last five years, experiments on angular scales from several degrees to a few minutes have started to delineate the CMB power spectra (see Smoot (1997) for a recent review), offering a unique view in astronomy on the conditions prevailing in the early Universe and the opportunity to determine the most important cosmological parameters. The current observations put constraints on the level of normalization of the Sachs-Wolfe effect, and appear to establish the existence of the first acoustic peak (Hancock et al. 1998). However, the small amplitude of the CMB anisotropy $\left(\Delta T / T \sim 10^{-5}\right)$ and the necessity to measure it within a precision of $\Delta T / T \sim 10^{-6}$ to determine the cosmological parameters with uncertainties $\simeq 10 \%$, is in the limit of the sensitivity of current technology and a challenge for the next generation of CMB experiments. Here, we present the results of the Tenerife experiments at 10 and $15 \mathrm{GHz}$ in a sky region covering $\sim 5000$ and $\sim 6500$ square degrees respectively of which we have selected $\sim 2000$ square degrees lying at high Galactic latitude. The multifrequency nature of the experiment allows a separation of possible contributions of the diffuse Galactic emission and other foregrounds from the CMB fluctuations. The observations presented here are sensitive to scales $\sim 2^{\circ}-6^{\circ}$ (the Sachs-Wolfe regime of the CMB power spectra). This angular range can be used to test the inflationary models, to measure directly the level of normalization, the spectral index of the primordial fluctuations and the possible existence of a foreground of gravitational waves. The paper is organized as follows: Section 2 presents a brief summary of the observations and data processing, Section 3 presents the maps of the sky at both frequencies. The contribution of foregrounds is considered in Section 4. Section 5 analyses statistically the properties of the signals detected, while Section 6 summarises the results and brings together the conclusions reached.

\section{OBSERVATIONS}

The Tenerife CMB experiments are a collaboration between the University of Manchester, the Instituto de Astrofísica de Canarias (IAC) and the Mullard Radio Astronomy Observatory (MRAO) which started in the year 1984 with the objective to detect and map the CMB structure generated by the Sachs-Wolfe effect. The observations are conducted by three drift scanning radiometers at 10, 15 and $33 \mathrm{GHz}$ installed at The Teide Observatory in Tenerife (Spain). The instruments have two independent receiver channels and operate using a double-difference technique, with a beam response of the form $-0.5,+1,-0.5$ (the positive in the meridian and the other two displaced $8^{\circ} 1$ in right ascension) and a primary beam of full width half maximum $(\mathrm{FWHM}) \sim 5^{\circ}$. The instruments are sensitive to a range in multipoles $(\ell \sim 10-30)$ which 
corresponds roughly to angular scales on the sky of $\theta \sim 2^{\circ}-6^{\circ}$. A detailed description of the instruments and observing strategy can be found, for example, in Davies et al. (1996).

This paper presents the data taken by the experiments at 10 and $15 \mathrm{GHz}$ until the end of 1997 . The instruments observe strips of the sky separated by 2.5 , this separation (half of the FWHM) fully samples the region between adjacent declinations allowing the reconstruction of a map of the sky fluctuations at each frequency. The strips analysed here range from Decs. $=32.5^{\circ}$ to $42.5^{\circ}$ at $10 \mathrm{GHz}$, and from Decs. $=+27.5^{\circ}$ to $+45^{\circ}$ at $15 \mathrm{GHz}$. We have not included data from the $33 \mathrm{GHz}$ radiometer as the sky coverage is still sparse as compared to the other two experiments. Figure 1 presents the sky region observed in a Mollweide projection; the ring represents full strips in RA with the inner and outer edges corresponding to Dec. $=+27.5^{\circ}$ and $+45^{\circ}$ respectively (the $15 \mathrm{GHz}$ data). The analysis presented here is limited to the section of our data at high Galactic latitude $\left(|b| \gtrsim 40^{\circ}\right)$. The pre-processing (not presented in this paper) includes calibration, discarding data closer than $50^{\circ}$ and $30^{\circ}$ to the Sun and Moon respectively and the removal of long term (on timescales of several hours) atmospheric baselines simultaneously with the Maximum Entropy analysis used to reconstruct the intrinsic sky signal (see Jones et al. 1998). The surviving data in each position of the sky are stacked together in order to reduce the level of the noise. These stacked second-difference data at high Galactic latitude at each declination and frequency are shown in Figure 2. Table 1 presents a summary of the sensitivity achieved in a beam size $\left(5^{\circ}\right)$ and the average number of individual measurements per pixel contributing in each of these declination strips. At $10 \mathrm{GHz}$ the coverage is nearly uniform in terms of noise and number of observations; however at $15 \mathrm{GHz}$ the coverage is less uniform with the poor declination being Dec. $=+27.5^{\circ}$ which is twice as noisy as the rest of the scans. From the table, it is possible to compute the mean sensitivity on a single $1^{\circ}$. measurement, obtaining $\sim 480$ and $225 \mu \mathrm{K}$ at 10 and $15 \mathrm{GHz}$ respectively. This difference in sensitivity is intrinsic to the instruments, being mainly a result of the difference in the bandwidth in frequency of the detectors $(\sim 0.5$ and $\sim 1.5 \mathrm{GHz}$ for the receptors at 10 and $15 \mathrm{GHz}$ respectively).

\section{MAPS AT 10 AND 15 GHZ}

The data presented in Figure 2 represent the intrinsic signal on the sky convolved with the triple beam response of the experiment and with the addition of baselines and noise. To obtain the intrinsic signal on the sky some method of deconvolution is needed; several techniques have been applied to astronomical data: CLEAN, the Wiener filter (Bunn, Hoffman and Silk, 1996) and the Maximum Entropy Method 
Table 1: Sensitivity of the data at 10 and $15 \mathrm{GHz}$

\begin{tabular}{ccccc} 
Dec. $\left(^{\circ}\right)$ & \multicolumn{2}{c}{$10 \mathrm{GHz}$} & \multicolumn{2}{c}{$15 \mathrm{GHz}$} \\
\hline & Num & $\sigma(\mu \mathrm{K})$ & Num. & $\sigma(\mu \mathrm{K})$ \\
\hline 27.5 & & & 48 & 35 \\
30.0 & & & 151 & 18 \\
32.5 & 76 & 58 & 120 & 21 \\
35.0 & 74 & 56 & 160 & 18 \\
37.5 & 81 & 51 & 96 & 21 \\
40.0 & 95 & 50 & 130 & 22 \\
42.5 & 66 & 54 & 84 & 23 \\
45.0 & & & 83 & 23
\end{tabular}

(MEM) (White \& Bunn (1995), Jones et al. 1998). The MEM method has been applied to the Tenerife CMB data $10 \mathrm{GHz}$ data with the old instrumental configuration ( $8^{\circ} \mathrm{FWHM}$ ). The method allows for the reconstruction of positive and negative features by considering the intrinsic CMB structure as the difference of two positive additive distributions as explained in Maisinger, Hobson, \& Lasenby (1997). The details of the method and its implementation for the analysis of the Tenerife data can be found in Jones et al. (1998). The only differences with that work are the beam size of the instruments ( $5^{\circ} \mathrm{FWHM}$ ) instead of $8^{\circ} \mathrm{FWHM}$ in the old configuration, and that now the contribution of the point sources is subtracted in each of the observations before the MEM reconstruction. The estimation of this contribution is explained in Section 4.

We applied the MEM deconvolution to the 10 and $15 \mathrm{GHz}$ data independently; an extension of the technique with a joint analysis of data taken at several frequencies is now in progress and will be presented in a future paper. The sampling of the observations ( $1^{\circ}$ in RA and $2.5^{\circ}$ in Dec.) is enough to fully sample the sky. The main feature on the observed sky region is the emission from the Galactic plane with amplitudes as large as $60 \mathrm{mK}$ for the major crossing at $10 \mathrm{GHz}$ in the strip at Dec. $=+40^{\circ}$ (Gutiérrez et al. 1995). These amplitudes are several order of magnitudes larger than the expected amplitudes due to the contribution of discrete radio sources, the diffuse emission at high Galactic latitudes, and the CMB fluctuations. As was pointed out in the paper by Jones et al. (1998), this large dynamical range prevents the simultaneous reconstruction of both the dominant features due to the Galactic plane crossing and the 
features lying at high Galactic latitudes. The reconstruction presented here is limited to the section of our observations at high Galactic latitudes $|b| \gtrsim 40^{\circ}$.

Figure 3 shows the maps of the sky reconstruction at 10 and $15 \mathrm{GHz}$ after subtraction of the discrete point-source contribution (see next section). The errorbars were calculated as a variance over 300 Monte-Carlo reconstructions of the same sky with different noise realisations giving values of $46 \mu \mathrm{K}$ and 35 $\mu \mathrm{K}$ per $1^{\circ}$ square pixel at 10 and $15 \mathrm{GHz}$ respectively, or $\sim 8$ and $\sim 6 \mu \mathrm{K}$ respectively in a beam-size region $\left(5^{\circ} \times 5^{\circ}\right)($ compared with $31 \mu \mathrm{K}$ and $12 \mu \mathrm{K}$ in the raw data).

The two MEM processed maps can be compared to each other to discern the origin of the fluctuations present. Features present in both the $10 \mathrm{GHz}$ and $15 \mathrm{GHz}$ reconstructed maps are likely to be $\mathrm{CMB}$ in origin whereas features that appear with a greater amplitude in the $10 \mathrm{GHz}$ reconstruction are likely to be Galactic in origin. It is important to note that the greyscales on the two maps are not the same so that a $\mathrm{CMB}$ feature will appear less bright (by a factor of 2) in the $10 \mathrm{GHz}$ map than in the $15 \mathrm{GHz} \mathrm{map}$.

The two most obvious CMB features that appear in both maps are at Dec. $40^{\circ}$, RA $180^{\circ}$ and Dec. $35^{\circ}, \mathrm{RA} 220^{\circ}$. Both appear with an amplitude of about $200 \mu \mathrm{K}$ in both maps and are therefore very likely to be $\mathrm{CMB}$ in origin. These are in agreement with the results of the comparison between the $\mathrm{COBE}$ DMR and the Tenerife data (Lineweaver et al. (1995), Gutiérrez et al. 1997). Possible Galactic sources (corresponding to either synchrotron or free-free emission) seen in the $10 \mathrm{GHz}$ map and at about half the amplitude in the $15 \mathrm{GHz}$ map are at Dec. $40^{\circ}$, RA $210^{\circ}$ and Dec. $37.5^{\circ}$, RA $240^{\circ}$ (which was also seen at $10 \mathrm{GHz}$ in the 8 degree switched beam reported by Jones et al., (1998)). There are numerous other features that are unaccounted for which appear in the two maps. Some may be due to noise or residual point sources but others may be Galactic or CMB in origin that the present data has not been able to constrain very accurately. For example, the feature which just appears at the bottom of the $10 \mathrm{GHz}$ map (Dec. $32.5^{\circ}$, RA $180^{\circ}$ ) and appears in the $15 \mathrm{GHz}$ map as an extended source at slightly lower declination and RA may be Galactic in origin. This source has been detected in the Jodrell Bank 5 GHz survey (Jones et al. (1999)b) with an amplitude implying a Galactic spectral index (between 2 and 3) between $5 \mathrm{GHz}$ and $15 \mathrm{GHz} . \mathrm{A}$ more comprehensive joint analysis of the two data sets allowing a seperation of the CMB and Galactic components will be presented in a later paper.

\section{FOREGROUNDS}




\subsection{Point sources}

The contribution of the discrete point sources has been estimated from the measurements by the Michigan monitoring programme (Aller \& Aller 1997); these have been complemented by the Kühr et al. (1981) and Green Bank (Condon, Broderick \& Seielstad 1989) catalogues of discrete radio sources. Our estimation for this foreground takes into account the time variability of each source and represents an improvement over previous estimations using just the Kühr et al. and Green Bank catalogues. Given the beam size of the instruments $\left(5^{\circ} \mathrm{FWHM}\right)$ the contribution of any point like source is highly diluted in our data, for instance a source with a flux of $1 \mathrm{Jy}$ contributes 35 and $12 \mu \mathrm{K}$ at 10 and $15 \mathrm{GHz}$ respectively. In the section of our data at high Galactic latitudes the main contributors are the sources $3 \mathrm{C} 345\left(\mathrm{RA}=16^{h} 41^{m} 18^{s}\right.$, Dec. $\left.=39^{\circ} 54^{\prime} 11^{\prime \prime}\right), 4 \mathrm{C} 39.25\left(\mathrm{RA}=9^{h} 23^{m} 56^{s}\right.$ Dec. $\left.=39^{\circ} 15^{\prime} 23^{\prime \prime}\right)$ and $3 \mathrm{C} 286\left(\mathrm{RA}=13^{h} 28^{m} 50^{s}\right.$, Dec. $\left.=30^{\circ} 45^{\prime} 59^{\prime \prime}\right)$. Of the three mentioned sources only $3 \mathrm{C} 286$ does not show significant time variability. The other two sources change over the observing period by factors of $2-3$, which makes it essential to have time-dependent data for them. In general, sources with smaller fluxes also show similar relative time variability, but their effect on our measurements is not significant.

We have taken the measurements by Aller \& Aller at 4.8, 8.0 and $14.5 \mathrm{GHz}$; which include all the sources in our observing region with amplitudes $\gtrsim 1 \mathrm{Jy}$. We fitted a polynomial to the flux variability at each frequency and checked the consistency of this fit with the predictions (Gutiérrez et al. 1999). For each month we computed the corresponding flux at our observing frequencies which was then converted into antenna temperature and convolved with the beam of our experiments to reproduce the contribution of such sources in our data. The rms of our data due to the contribution of these discrete radio sources in the region $\mathrm{RA}=130^{\circ}-260^{\circ}$ lies between $\sim 40$ and $\sim 120 \mu \mathrm{K}$, and between $\sim 15$ and $\sim 40 \mu \mathrm{K}$ at 10 and $15 \mathrm{GHz}$ respectively in a bin of $1^{\circ}$ in $\mathrm{RA}$; the larger rms occurs in the scan at Dec. $=+40^{\circ}$ and is due mainly, as has been explained above, to the contribution of the sources $4 \mathrm{C} 39.25$ and $3 \mathrm{C} 345$. The contribution due to a foreground of unresolved radio-sources is expected to have an rms $\lesssim 30$ and $\lesssim 15 \mu \mathrm{K}$ at 10 and $15 \mathrm{GHz}$ respectively (Franceschini et al. 1989).

Figure 4 shows the comparison between the predicted contribution of the main radio sources (3C 345, 3C39.25 and 3C 286) and our measurements. At $15 \mathrm{GHz}$ the peak contribution of the sources is in the range $70-120 \mu \mathrm{K}$, while at $10 \mathrm{GHz}$ the range is $300-400 \mu \mathrm{K}$. The agreement between the prediction of the contribution of these radio sources and our measurements represents an important check on the consistency of our data. 


\subsection{Diffuse Galactic component}

The most direct approach to analyse the possible diffuse Galactic component is by using low frequency surveys, like the ones at $408 \mathrm{MHz}$ (Haslam et al. 1982) and $1420 \mathrm{MHz}$ (Reich \& Reich 1988). However, there are several papers showing the unreliability of these two surveys when used to predict this contribution for CMB experiments working at centimeter wavelengths (for example, Davies, Watson \& Gutiérrez 1996). Additional problems for these predictions come from the fact that neither the frequency spectral index or the spatial power spectra of the diffuse Galactic emission is well known. Existing evidence shows a steepening of this frequency spectral index with frequency. For instance Bersanelli et al. (1996) found a value $2.9 \pm 0.3$ between 1.42 and $5 \mathrm{GHz}$ at angular scales $\sim 2^{\circ}$. At larger angular scales Platania et al. (1997) obtained a spectral index for the synchrotron emission near the Galactic plane of $2.81 \pm 0.16$ in the range 1.0-7.5 GHz, with evidence of steepening at frequencies above $7.5 \mathrm{GHz}$.

The limitations mentioned above on the low frequency surveys have motivated the development of alternative methods to separate this foreground from the CMB structure. One of these will be presented in a forthcoming paper (Jones et al. (1999) $a$ in preparation) in which the Tenerife data at 10, 15 and $33 \mathrm{GHz}$, and the COBE DMR data (Bennett et al. 1996) are incorporated in an extension of the MEM method reconstructing the most probable $\mathrm{CMB}$ and Galactic maps. Other analyses which cross-correlate sky surveys at centimeter and millimeter wavelengths with the data presented here, are also in progress. In

this work we have made a joint likelihood analysis of the data at 10 and $15 \mathrm{GHz}$. This method provides by itself a statistical way to separate the CMB and the Galactic component; this is explained in next section.

\section{STATISTICAL ANALYSIS}

\subsection{Likelihood analysis}

In standard inflationary models the CMB fluctuations are a realization of a random gaussian field, and the probability distribution is fully determined by the angular power spectrum or alternatively by the Legendre transformation, the two-point correlation function. This can be determined using a likelihood analysis. Methods based on the likelihood function have been extensively used in the analysis of CMB data (see for example Hancock et al. (1997) for a general discussion, and Gutiérrez et al. (1995) for the application of the method to the Tenerife data). The method computes by a Bayesian inference the statistical probability distribution of the sky signal, considering the correlation present between each pair 
of data points and taking into account the observing strategy, experimental configuration, properties of the noise, etc. Experimental data are the result of convolution of the sky signal with the beam response of the instruments, with the addition of some noise. This response can be characterized by the experimental window function $W_{\ell}$ which defines the filtering produced by the experiment at each angular mode of the power spectrum. In the case of the Tenerife experiments the window function is sensitive to a range in mutipoles $\ell \sim 10-30$ with a maximum response to $\ell \sim 20$. In addition to this filtering, the observed correlation function contains the contribution from the noise. The likelihood function then follows a multinormal distribution with the covariance matrix composed of two terms, one due to the signal and one due to the noise: $\mathbf{C}=\mathbf{S}+\mathbf{N}$. In our case the data consist of a set of second differences in temperature at 10 and $15 \mathrm{GHz}$, along with their errors, binned at $1^{\circ}$ in RA. For the matrix of the noise, the only non-zero terms are those on the diagonal, as the instrumental noise is uncorrelated in scales larger than the sampling in RA (Gutiérrez et al. 1997). The method assumes a given model for the sky signal with a free parameter (the level of normalization) which will be constrained according to its compatibility with the data.

We have applied the likelihood analysis to the data at $10 \mathrm{GHz}$ and $15 \mathrm{GHz}$ after subtraction of the discrete point-source contribution. We assumed a model for the sky signal in which the contribution by each multipole to the total power spectrum is the same for all multipoles at which the experiment is sensitive; these kind of models are called flat band power spectrum. We restricted the analysis to sections of data in the observing region $\mathrm{RA}=120^{\circ}-270^{\circ}$ which is away from the Galactic plane. Table 2 summarizes the results. The quantity quoted is $\Delta T_{\ell}=\sqrt{\ell(\ell+1) C_{\ell} / 2 \pi}$. The table also shows the value of the likelihood peak relative to the value in the absence of signal, which shows the high significance of the detected signal. We see that including the section $\mathrm{RA}=230^{\circ}-250^{\circ}$ the signal detected is slightly larger. For the estimation of the $\mathrm{CMB}$ signal we are more confident on the results in the range $\mathrm{RA}=161^{\circ}-230^{\circ}$ which excludes possible residuals due to the contribution of the variable sources $3 \mathrm{C} 345$ and $4 \mathrm{C} 39.25$.

We have performed the same analysis for the $10 \mathrm{GHz}$ data. In the analysis of each scan separately there is no clear evidence of detection in the RA range $120^{\circ}-260^{\circ}$ except for the scan at Dec. $=+32.5^{\circ}$ which has a detection of signal at the two-sigma level. The simultaneous analysis of the 5 scans gives the result shown in Table 2. The detection here is compatible with the results at $15 \mathrm{GHz}$, but less significant as a consequence of the higher noise and less sky coverage at each frequency as compared with the data at 15 GHz. 
Table 2: Likelihood results at 10 and $15 \mathrm{GHz}$

\begin{tabular}{lcccc}
\hline Data & Dec. $\left(^{\circ}\right)$ & $\mathrm{RA}\left({ }^{\circ}\right)$ & $\Delta T_{\ell}(\mu \mathrm{K})$ & $L_{\max }$ \\
\hline $15 \mathrm{GHz}$ & $27.5-45$ & $141-210$ & $35_{-7}^{+8}$ & $3.2 \times 10^{10}$ \\
$15 \mathrm{GHz}$ & $27.5-45$ & $141-230$ & $35_{-6}^{+8}$ & $1.3 \times 10^{15}$ \\
$15 \mathrm{GHz}$ & $27.5-45$ & $141-250$ & $39_{-7}^{+7}$ & $2.6 \times 10^{29}$ \\
$15 \mathrm{GHz}$ & $27.5-45$ & $161-230$ & $30_{-7}^{+9}$ & $1.7 \times 10^{8}$ \\
$15 \mathrm{GHz}$ & $27.5-45$ & $161-250$ & $37_{-6}^{+8}$ & $2.1 \times 10^{22}$ \\
$15 \mathrm{GHz}$ & $27.5-45$ & $181-250$ & $32_{-6}^{+8}$ & $1.6 \times 10^{19}$ \\
\hline \hline $10 \mathrm{GHz}$ & $32.5-42.5$ & $141-210$ & $\leq 47$ & 1.6 \\
$10 \mathrm{GHz}$ & $32.5-42.5$ & $141-230$ & $\leq 35$ & 1.1 \\
$10 \mathrm{GHz}$ & $32.5-42.5$ & $141-250$ & $29_{-10}^{+10}$ & 57.0 \\
$10 \mathrm{GHz}$ & $32.5-42.5$ & $161-230$ & $\leq 30$ & 1.0 \\
$10 \mathrm{GHz}$ & $32.5-42.5$ & $161-250$ & $27_{-11}^{+11}$ & 19.7 \\
$10 \mathrm{GHz}$ & $32.5-42.5$ & $181-250$ & $27_{-11}^{+12}$ & 17.8
\end{tabular}

Table 3: Likelihood results of the splits at 10 and $15 \mathrm{GHz}$

\begin{tabular}{lcccc}
\hline Data & Dec. $\left(^{\circ}\right)$ & $\mathrm{RA}\left(^{\circ}\right)$ & $\Delta T_{\ell}(\mu \mathrm{K})$ & $L_{\max }$ \\
\hline $15 \mathrm{GHz} \mathrm{A}$ & $27.5-45$ & $161-230$ & $31_{-8}^{+9}$ & $9.4 \times 10^{4}$ \\
$15 \mathrm{GHz} \mathrm{B}$ & $27.5-45$ & $161-230$ & $27_{-7}^{+9}$ & $1.1 \times 10^{4}$ \\
$15 \mathrm{GHz}(\mathrm{A}-\mathrm{B}) / 2$ & $27.5-45$ & $161-230$ & $\leq 13$ & 1.2 \\
\hline \hline $10 \mathrm{GHz} \mathrm{A}$ & $32.5-42.5$ & $161-230$ & $<54$ & 1.1 \\
$10 \mathrm{GHz} \mathrm{B}$ & $32.5-42.5$ & $161-230$ & $<59$ & 1.6 \\
$10 \mathrm{GHz}(\mathrm{A}-\mathrm{B}) / 2$ & $32.5-42.5$ & $161-230$ & $\leq 34$ & 1.0
\end{tabular}




\subsubsection{JOINT ANALYSIS}

The first model considered in the joint likelihood analysis of the 10 and $15 \mathrm{GHz}$ data assumes that the same signal is present in the data at both frequencies; this excludes a priori the possibility to have any signal changing with frequency between 10 and $15 \mathrm{GHz}$. For the reason mentioned above, we limited the analysis to the section of data at $\mathrm{RA}=161^{\circ}-230^{\circ}$. Analyzing the five common strips at 10 and $15 \mathrm{GHz}$ we obtain a signal $\Delta T_{\ell}=30_{-8}^{+10} \mu \mathrm{K}$ with a likelihood peak of $1.5 \times 10^{6}$. Comparing these with the results of the analysis of each frequency separately, we see that the signal is similar to that found at 10 and 15 GHz. This indicates the compatibility between the data at both frequencies (i.e. the signal present in both frequency has not only the same amplitude but also comes from the same structures). However, this agreement is not complete, as suggested by the shape of the likelihood function which is broader than the likelihood function analyzing the $15 \mathrm{GHz}$ alone.

Two observing frequencies potentially allows the separation of the CMB and Galactic components. The likelihood analysis offers a natural method to distinguish between signal with and without spectral dependance (Gorski et al. 1996). We assume that the signals in the data at 10 and $15 \mathrm{GHz}$ are given by $\Delta T_{\ell}^{10,15}=\Delta T_{\ell_{C M B}}+\Delta T_{\ell_{G A L}}^{10,15}$, where $\Delta T_{\ell_{G A L}}^{15}=\Delta T_{\ell_{G A L}}^{10} \times(14.9 / 10.4)^{\alpha}$, and $\alpha$ is the spectral index of the Galactic emission. The model considers that the CMB signal is characterized by a flat power spectra along the window function of the experiment, while the power spectra of the diffuse Galactic component is given by $\Delta T_{\ell_{G A L}} \propto \ell^{-k}$ with $k=0.5$ (Gautier et al. (1992), Bersanelli et al. 1996) which means a change in the Galactic contribution by a factor $\sim \sqrt{3}$ along the window function of the experiment. We have chosen values of -2.15 and -3.0 for the spectral index of the Galactic component which correspond to two cases in which this contribution is dominated by free-free and synchrotron emission respectively. Analyzing the common strips at 10 and $15 \mathrm{GHz}$ in the $\mathrm{RA}$ range $161^{\circ}-230^{\circ}$ we obtain $\Delta T_{\ell_{C M B}}=30_{-11}^{+15} \mu \mathrm{K}$ and $\Delta T_{\ell_{G A L}}^{10}<28 \mu \mathrm{K}$ (68\% C. L.) for the possible free-free component at $10 \mathrm{GHz}$; similar results are obtained assuming a synchrotron component. This value is our best estimation of the CMB signal detected by the Tenerife experiments. There is no detection of any Galactic component in any of the cases considered. As a result the CMB signal has the same amplitude as in the previous pure CMB models. As expected the uncertainty associated with the CMB signal is larger now as a consequence of the new free parameter in the analysis.

Fig. 7 presents this likelihood function. The results indicate the consistency of the data at 10 and $15 \mathrm{GHz}$, and that the level of the Galactic contamination is below the sensitivity of our data. 
Assuming a Harrison-Zeldovich spectrum for the primordial fluctuations, the value corresponds to an expected quadrupole $Q_{R M S-P S}=20_{-7}^{+10} \mu \mathrm{K}$ (68\% C.L.). This value includes the uncertainties due to the instrumental error, the sample variance and the possible existence of an undetected diffuse Galactic component. This result is in agreement with $Q_{R M S-P S}=18 \pm 1.6 \mu \mathrm{K}$, the value of the COBE DMR normalization (Bennett et al. 1996), and implicitly demonstrates the validity of the Harrison-Zeldovich model in the range of multipoles covered by the COBE and Tenerife experiments $(l=2-30)$.

\subsection{SPLITS OF THE DATA}

To check the consistency of the results we have split the data at 10 and $15 \mathrm{GHz}$ into two halves $A$ and $B$ with similar sensitivities, and compute the sum $(A+B) / 2$ and the difference $(A-B) / 2$. If both halves are consistent they should contain the same signal while the difference should contain just pure noise. We carried out the likelihood analysis of these obtaining the results shown in Table 3. At $15 \mathrm{GHz}$ this analysis indicates the clear presence of signals with similar amplitudes in both halves. The difference $(A-B) / 2$ does not show the presence of any signal, being compatible with noise. The likelihood curves of the $A, B$, $(A+B) / 2$ and $(A-B) / 2$ data are plotted in Figure 5 . As expected at $10 \mathrm{GHz}$, the splits $A$ and $B$, and the difference $(A-B) / 2$ are compatible with noise, due to the lack of sensitivity and the consistency between both halves respectively.

An additional test has been done by computing the experimental cross-correlation between the two halves $A$ and $B$ at $15 \mathrm{GHz}$, and comparing it with the prediction for a given theoretical model. In our case the beam response is not rotationally invariant due to the beam switching tecnhique along lines of constant declination; this makes the observed two-point correlation function depend on the separation in RA-Dec. Computing it along the separation in RA we obtain the correlation as a function of the separation in RA, $C\left(\theta_{i j}\right)=\sum_{i j} \Delta T_{i_{1} j_{1}} \Delta T_{i_{2} j_{2}} w_{i_{1} j_{1}} w_{i_{2} j_{2}} / \sum_{i j} w_{i_{1} j_{1}} w_{i_{2} j_{2}}$. Figure 6 (top) shows this experimental correlation function for the $15 \mathrm{GHz}$ data. At the bottom of Fig.6 it is represented the autocorrelation of the $(A-B) / 2$ (bottom) showing the compatibility between the data in the halves $A$ and $B$. The error bars in each case have been obtained by Monte Carlo techniques (1000 simulations). The solid line is the expected correlation assuming a signal given by a flat band power spectra with $\Delta T_{\ell}=30 \mu \mathrm{K}$ in the angular region of sensitivity of our experiment. The results obtained applying the same method to the $10 \mathrm{GHz}$ are not conclusive as a result of the larger noise in our data at this frequency. 


\section{CONCLUSIONS}

The Tenerife maps at 10 and $15 \mathrm{GHz}$ covering 5000 and 6500 square degrees on the sky centred around Dec. $+35^{\circ}$ have been presented; from these we have selected $\sim 2000$ square degrees at high Galactic latitude in which it is possible to analyze the properties of the CMB signal. The sensitivity achieved is enough to identify the most intense features. The strongest radio sources in the data at 10 and $15 \mathrm{GHz}$ have been identified at the level predicted. The data at $15 \mathrm{GHz}$ show the presence of a statistically very significant signal $\Delta T_{\ell}=30_{-7}^{+9} \mu \mathrm{K}$; a similar analysis for the $10 \mathrm{GHz}$ data show the compatibility with this signal, but the poor sensitivity means it is at the limit of detectability. A joint likelihood analysis of the 10 and $15 \mathrm{GHz}$ data allowing for the presence of a $\mathrm{CMB}$ and a Galactic signal gives values of $\Delta T_{\ell_{C M B}}=30_{-11}^{+15}$ $\mu \mathrm{K}$ and $\Delta T_{\ell_{G A L}}^{10}=<28 \mu \mathrm{K}$ (68\% C.L.). In the case of a Harrison-Zeldovich spectra for the primordial fluctutions, the CMB signal detected corresponds to $Q_{R M S-P S}=20_{-7}^{+10} \mu \mathrm{K}$ value in good agreement with the normalization at large angular scales provided by the COBE DMR data. Both results support the standard inflationary models.

The observations with the two radiometers continue in order to complete the sky region between Dec. $=+25^{\circ}$ and Dec. $=+45^{\circ}$ with uniform sensitivity. The data for the radiometer at $33 \mathrm{GHz}$ in the sky region between Dec. $=+35^{\circ}$ and Dec. $=+42.5^{\circ}$ are now under analysis. This extra spectral coverage will represent an important improvement to separate the diffuse Galactic component from the CMB signal as was shown in Hancock et al. (1994). The CMB observations from the Teide Observatory are being extended with several instruments working at frequencies between 10 and $33 \mathrm{GHz}$ and resolutions from a few degrees to $10^{\prime}$. The first of the new instruments is a $33 \mathrm{GHz}$ two-element interferometer (Melhuish et al. 1998) which has a resolution of 2.5 , with full sine and cosine correlation in a $3 \mathrm{GHz}$ bandwidth and which has obtained its first measurements at Dec. $=+41^{\circ}$ (Dicker et al. 1998). Additionally a set of three instruments with several bands centred at 10, 15 and $33 \mathrm{GHz}$ which are being built at the IAC will be installed during the year 1999. These instruments are designed to obtain a map of flux and polarization on a region of the sky between Dec. $=+10^{\circ}$ and Dec. $=+50^{\circ}$ with a resolution $\sim 1^{\circ}$. The collaboration between MRAO, NRAL and IAC continues with the Very Small Array (VSA). Operating at frequencies around $30 \mathrm{GHz}$ at the Tenerife site, the VSA will have the capability of imaging primordial CMB structure to a sensitivity of 5 $\mu \mathrm{K}$ over the angular range $10^{\prime}$ to $2^{\circ} .5$. This new set of instruments will provide a large spectral and angular coverage which will make it possible to delineate the CMB power spectra along the Sachs-Wolfe plateau and first few acoustic peaks, constraining some of the cosmological parameters at the $10 \%$ level. 


\section{Acknowledgments}

The Tenerife experiments are supported by the UK Particle Physics and Astronomy Research Council, the European Community Science programme contract SCI-ST920830, the Human Capital and Mobility contract CHRXCT920079 and the Spanish DGES science grant PB95-1132-C02. 


\section{REFERENCES}

Aller, M., \& Aller, H. 1997 (private communication)

Bennett, C. L. et al. 1996, ApJ, 464, L1

Bersanelli, M. 1996 (COBRAS/SAMBA report)

Bunn, E. F., Hoffman, Y., \& Silk, J. 1996, ApJ, 464, 1

Condon, J. J., Broderick, J. J. \& Seielstad, G. A. 1989, AJ, 97, 1064

Davies, R. D., Gutiérrez, C. M., Hopkins, J., Melhuish, S. J., Watson, R. A., Hoyland, R. J., Rebolo, R., Lasenby, A. N., \& Hancock, S. 1996, MNRAS, 278, 883

Davies, R. D., Watson, R. A., \& Gutiérrez, C. M. 1996, MNRAS, 278, 925

Dicker, S. R. et al. 1998, MNRAS (submitted)

Franceschini, A., Toffolatti, L., Danese, L., \& De Zotti, G. 1989, ApJ, 344, 35

Gautier, T. N., Boulanger, F., Perault, J. L. 1992, AJ, 103, 1313

Gorski, K. M. et al. 1996, astro-ph/9601063

Gutiérrez, C. M. 1997, ApJ, 483, 51

Gutiérrez, C. M. et al. 1999 (in preparation)

Gutierrez de la Cruz, C. M., Davies, R. D., Rebolo, R., Watson, R. A., Lasenby, A. N., \& Hancock, S. 1995 ApJ 442, 10

Hancock, S., R. D. Davies, Lasenby, A. N., C. M. Gutierrez de la Cruz, C. M., Watson, R. A., Rebolo, R., \& Beckman, J. E. 1994 Nature, 367, 333

Hancock, S., Gutierrez, C. M., Davies, R. D., Lasenby, A. N., Rocha, G., Rebolo, R., Watson, R. A., \& Tegmark, M. 1997 MNRAS, 289, 505

Hancock, S., Lasenby, A. N., Rocha, G., \& Gutiérrez, C. M. 1998, MNRAS, 294, 1

Haslam, C. G. T., Salter, C. J., Stoffel, H., \& Wison, W. E. 1982, AASS, 47, 1 
Jones, A. W., Gutiérrez, C. M., Mukherjee, P., Lasenby, A. N., Davies, R. D., Watson, R. A., \& Rebolo, R. 1999a, MNRAS (in preparation)

Jones, A. W., Hancock, S., Lasenby, A. N., Davies, R. D., Gutiérrez, C. M., Rocha, G., Watson, R. A., \& Rebolo, R. 1998, 294, 582

Jones, A. W., Wilkinson, A., Giardino, G., Melhuish, S. J., Asareh, H., Davies, R. D., \& Lasenby, A. N. 1999b (in preparation)

Lineweaver, C. H. et al. 1995, ApJ, 448, 482

Kühr, H., Witzel, A., Pauliny-Toth, I.I.K., \& Nauber, U. 1981, AASS, 45, 367

Maisinger, K., Hobson, M. P., \& Lasenby, A. N. 1997, MNRAS, 290, 313

Melhuish, S. J., Dicker, S., Davies, R. D., Gutierrez, C. M., Watson, R. A., Davis, R. J., Hoyland, R. J., \& Rebolo, R. 1998 MNRAS (submitted)

Platania, P. et al. 1997 astro-ph/9707252

Reich, P., \& Reich, W. 1988, AASS, 74, 7

Smoot, G. F. 1997 (astro-ph/9703084)

White, M., \& Bunn, E. 1995, ApJ, 443, L53 


\section{FIGURE CAPTIONS}

Figure 1. Mollweide projection of the sky showing the region observed by the Tenerife experiments.

Figure 2. The 10 and $15-\mathrm{GHz}$ stacked scans along with their errorbars; (left) $15 \mathrm{GHz}$ and (right) 10 GHz. The data have been binned in a $4^{\circ}$ bin in RA.

Figure 3. Maps at 10 and $15 \mathrm{GHz}$ obtained by the Maximum Entropy Method.

Figure 4. Comparison bewteen the measurements at 10 and $15 \mathrm{GHz}$ and the estimation of the contribution of the point sources 3C 345, 3C39.25 and 3C286.

Figure 5. Likelihood curves obtained splitting the data at $15 \mathrm{GHz}$ into two halves $A$ and $B$, and computing the sum $(A+B) / 2$ and the difference $(A-B) / 2$ respectively.

Figure 6. (Top) Croos-correlation of the splits $A$ and $B$ at $15 \mathrm{GHz}$ and the theoretical auto-correlation function for a Harrison-Zel'dovich spectrum, as convolved with the Tenerife beam and normalised to $Q_{r m s-p s}=20 \mu \mathrm{K}$. (Bottom) Autocorrelation of the $(A-B) / 2$.

Figure 7. Likelihood for the joint analysis of the 10 and $15 \mathrm{GHz}$ as a bivariate function of the amplitude of the Galactic and CMB signals. 


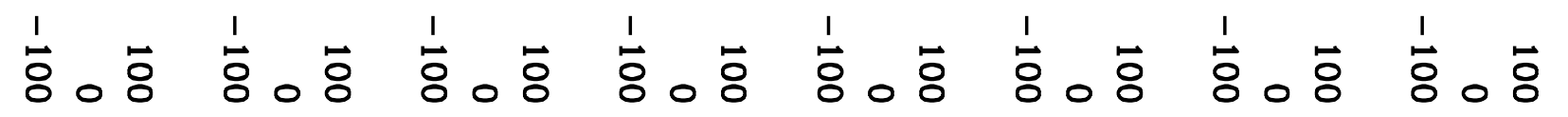

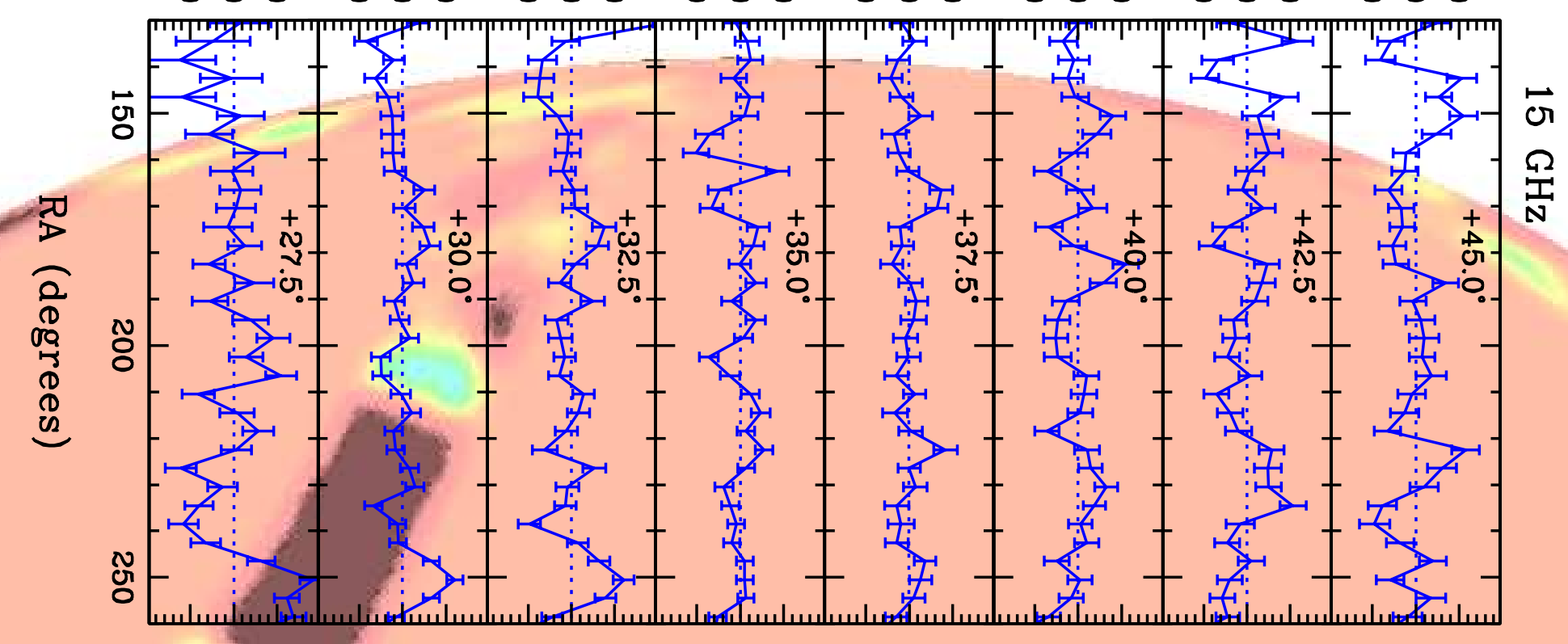

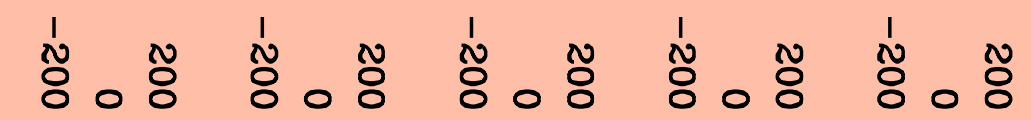

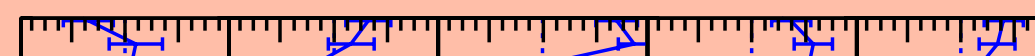

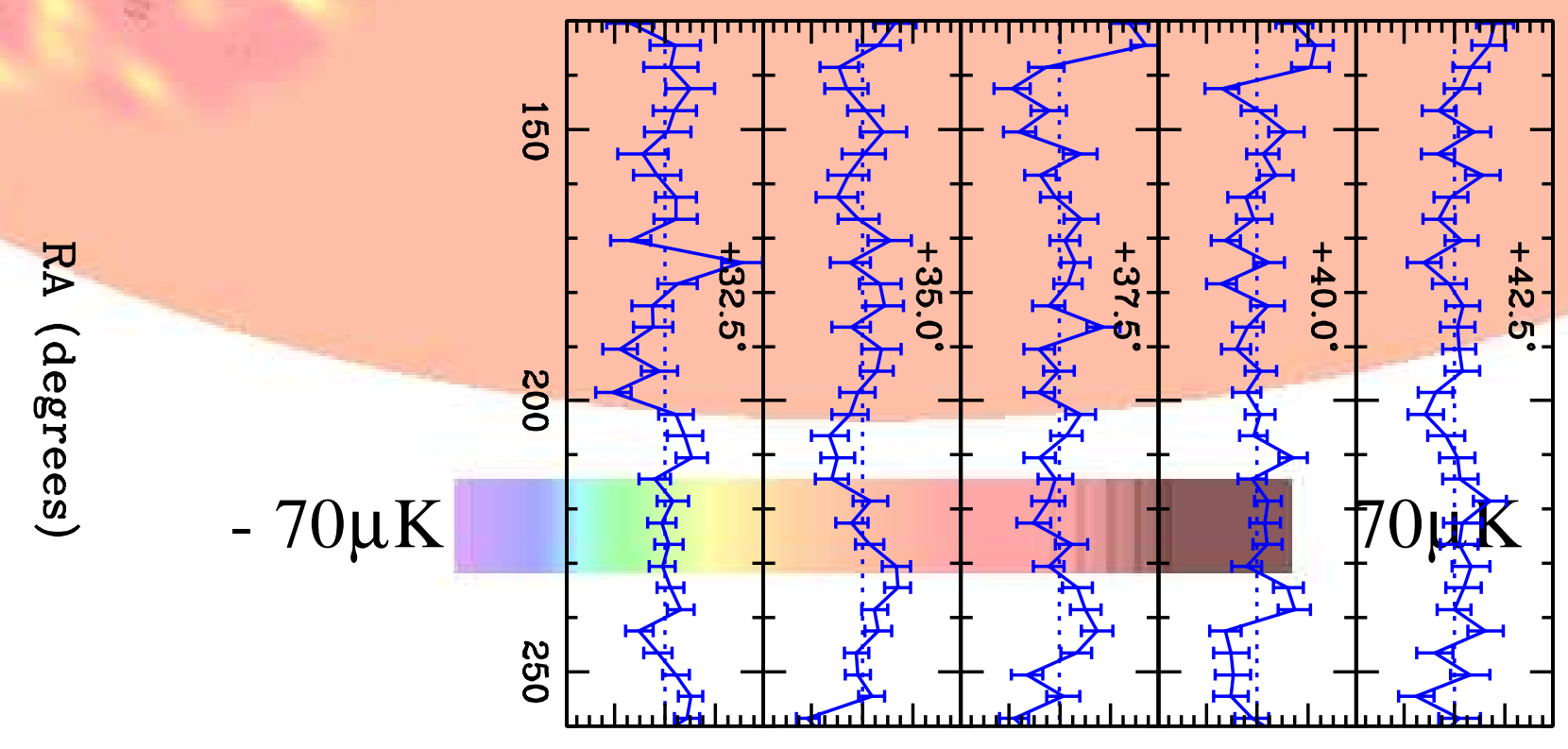

0 壱 

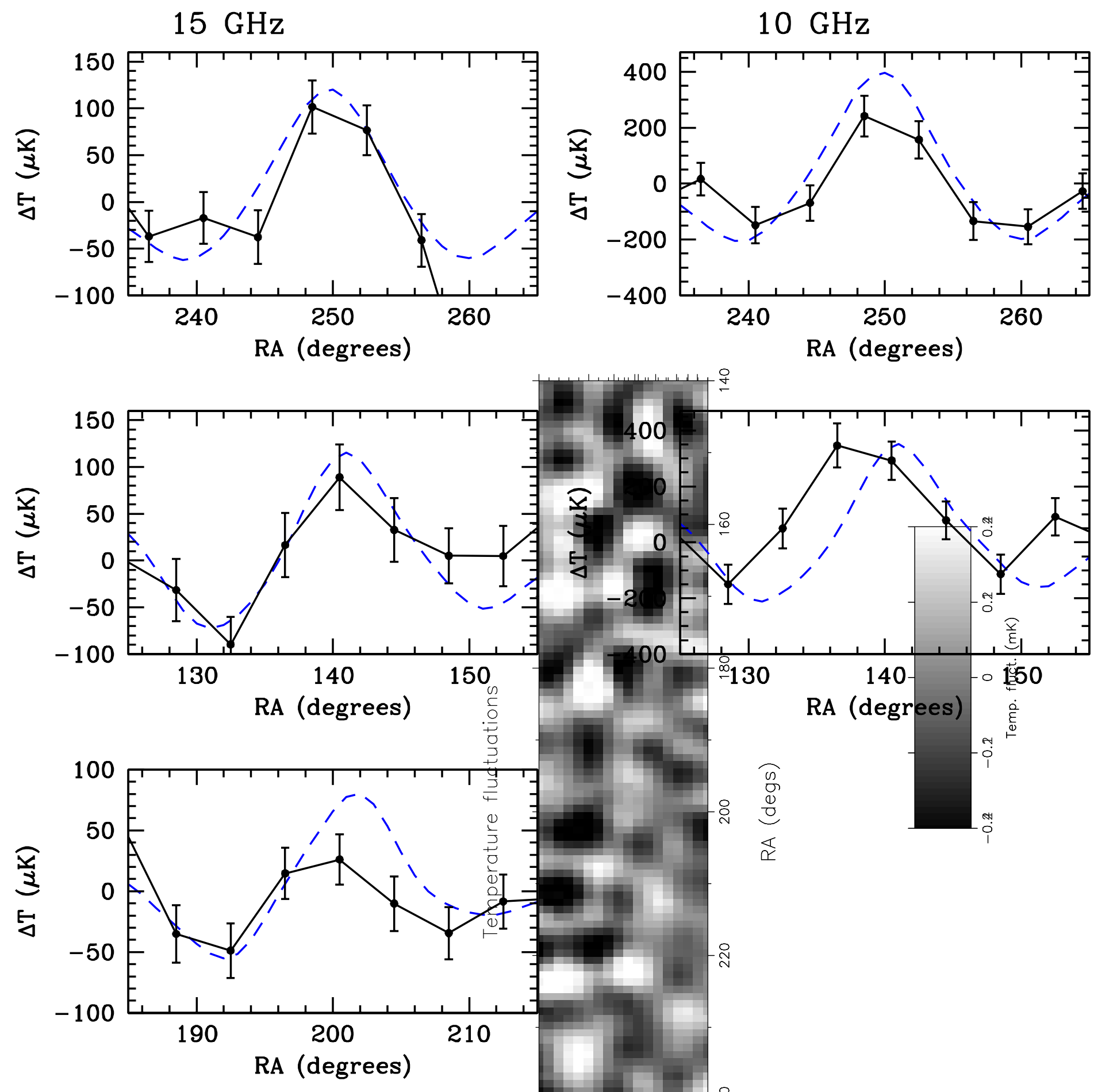
$15 \mathrm{GHz} \mathrm{A}$

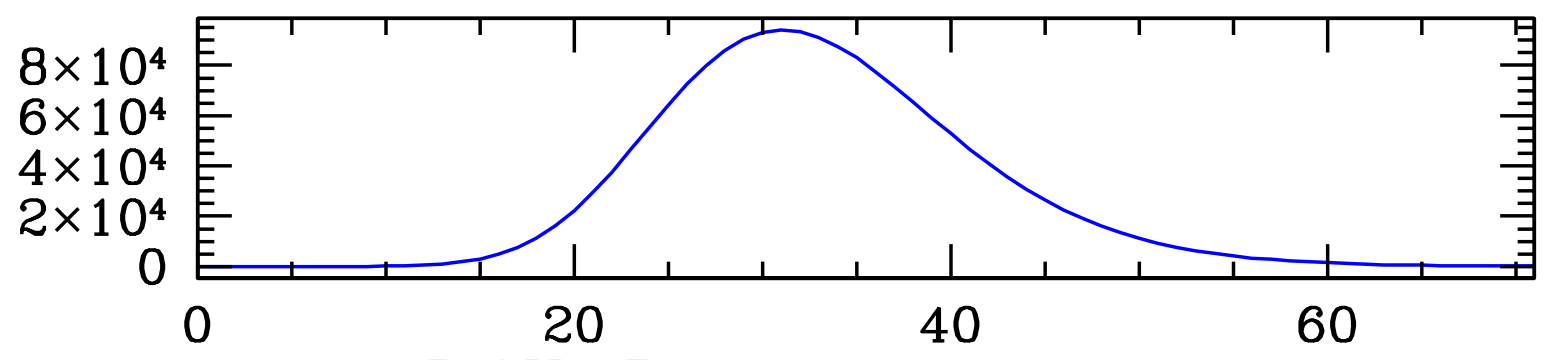

$15 \mathrm{GHz} B$
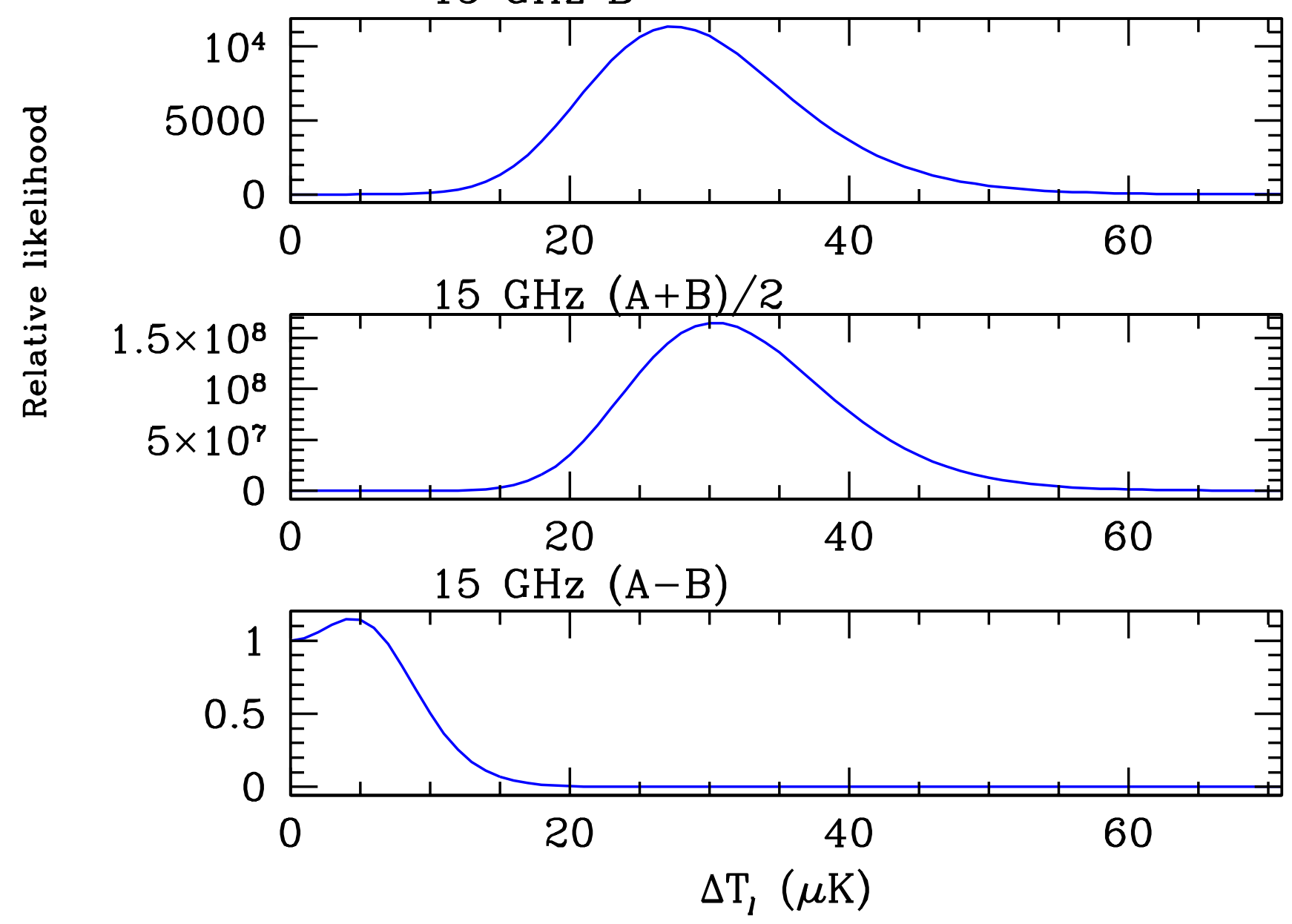


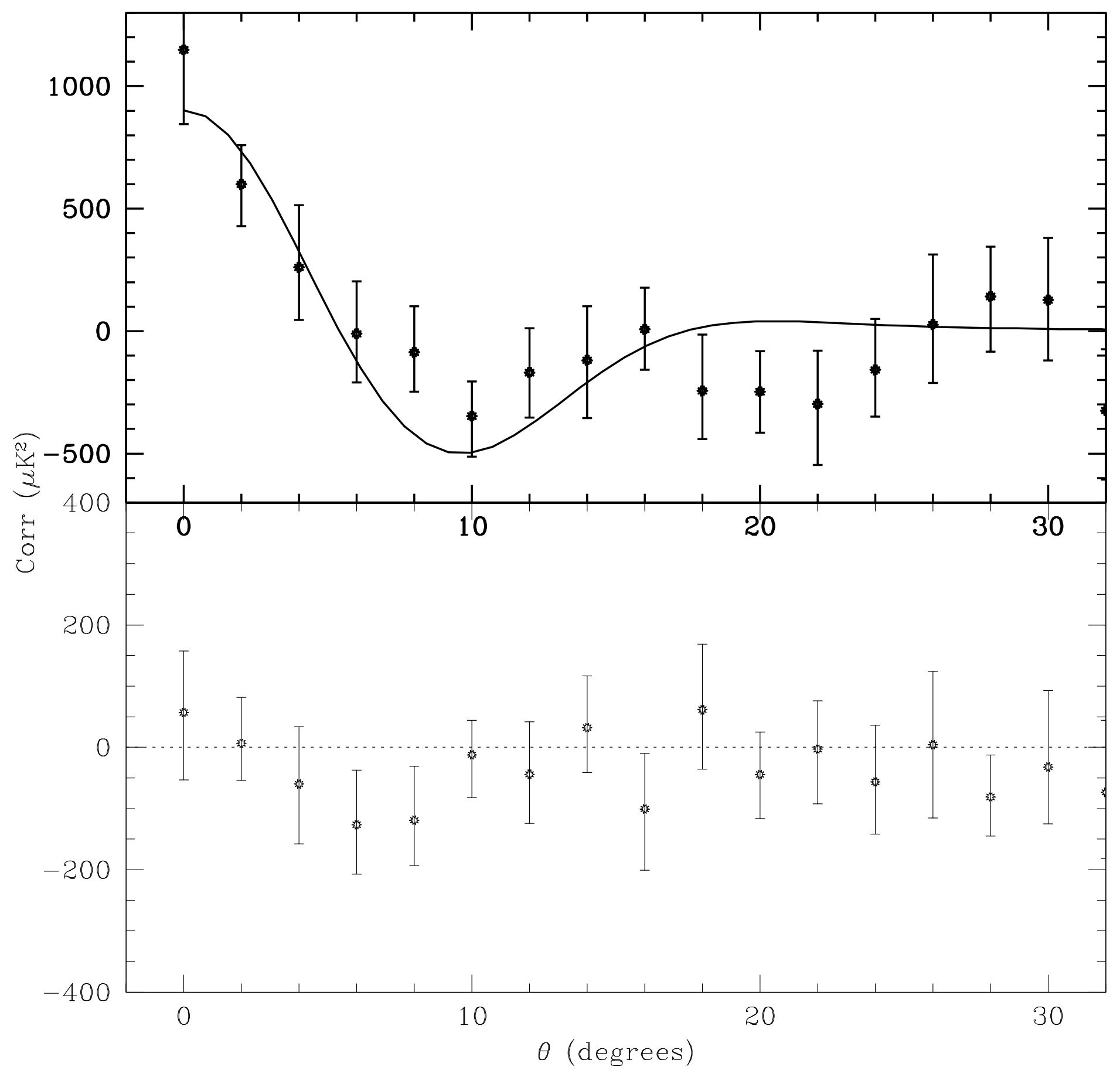

